\title{
DETC2021-70249
}

\section{PREDICTING MECHANICAL PROPERTIES OF 3D PRINTED LATTICE STRUCTURES}

\author{
Shuai $\mathrm{Ma}^{1,2}$ \\ Qian Tang ${ }^{1}$ \\ 1. State Key Laboratory of Mechanical Transmissions \\ Chongqing University \\ Chongqing, 400044, China
}

\author{
Ying Liü ${ }^{2} \quad$ Qixiang Feng ${ }^{1}$
}

2. Institute of Mechanical and Manufacturing Engineering

School of Engineering

Cardiff University

Cardiff, CF24 3AA, UK

\begin{abstract}
Lattice structures (LS) manufactured by $3 D$ printing are widely applied in many areas, such as aerospace and tissue engineering, due to their lightweight and adjustable mechanical properties. It is necessary to reduce costs by predicting the mechanical properties of LS at the design stage since $3 D$ printing is exorbitant at present. However, predicting mechanical properties quickly and accurately poses a challenge. To address this problem, this study proposes a novel method that is applied to different LS and materials to predict their mechanical properties through machine learning. First, this study voxelised $3 D$ models of the LS units and then calculated the entropy vector of each model as the geometric feature of the LS units. Next, the porosity, material density, elastic modulus, and unit length of the lattice unit are combined with entropy as the inputs of the machine learning model. The sample set includes 57 samples collected from previous studies. Support vector regression was used in this study to predict the mechanical properties. The results indicate that the proposed method can predict the mechanical properties of LS effectively and is suitable for different LS and materials. The significance of this work is that it provides a method with great potential to promote the design process of lattice structures by predicting their mechanical properties quickly and effectively.
\end{abstract}

Keywords: Lattice structures; mechanical properties; 3D printing; machine learning

\section{INTRODUCTION}

Lattice structures (LS), whether inspired by nature or created by mathematicians, are considered promising candidates for lightweight energy absorption and heat dissipation because their unique geometric shape can realise different functions. As FIGURE 1 shows, the most applied LS are body-centred cubic

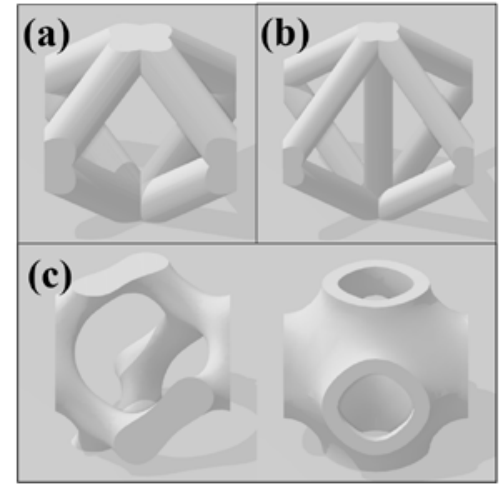

FIGURE 1: (a) BCC STRUCTURE UNIT; (b) BCCZ STRUCTURE UNIT; (c) TPMS STRUCTURE UNITS.

Additive manufacturing (AM), also called 3D printing, is an advanced manufacturing technology to fabricate complex parts that cannot be manufactured by traditional technology. AM includes various manufacturing methods, such as fused deposition modelling, electron beam melting, selective laser melting (SLM), and selective laser sintering. These methods also allow the manufacture of parts using non-metallic and metallic materials. SLM is widely applied in aerospace, automotive, and tissue engineering and moulds because it allows the use of many metallic powders: Ti6Al4V [5], stainless steel 316L [6], and maraging steel [7]. Furthermore, the layer-by-layer fabricated 
feature of SLM can freely manufacture samples with complex shapes and internal structures. Thus, SLM is considered a promising manufacturing method for fabricating metallic parts composed of LS.

The mechanical properties of LS are the basic requirements when they are used in various applications. LS have many advantages, and their elastic modulus and yield strength can be adjusted by designing with different unit parameters. This can save materials by choosing a suitable lattice structure to match the mechanical requirements. The elastic modulus is one of the most important mechanical properties of LS; it can achieve around $1 \%$ to $100 \%$ of the elastic modulus of solid material by manufacturing with different designed parameters.

In some application areas, the mechanical properties of parts composed of LS have strict design requirements. These include SLM-built bone scaffolds; as shown in FIGURE 2, the mechanical properties of the implanted scaffold should match those of damaged human bones to avoid "stress-shielding", which may lead to bone osteoporosis [8]. Furthermore, these kinds of porous scaffolds can also satisfy other functional requirements, such as good mass-transporting requirements [9].

Yield strength is another important mechanical property of parts composed of LS; parts will undergo permanent deformation if the loading stress is higher than yield strength. Thus, studying the yield strength of the LS can guide us to avoid parts failure.

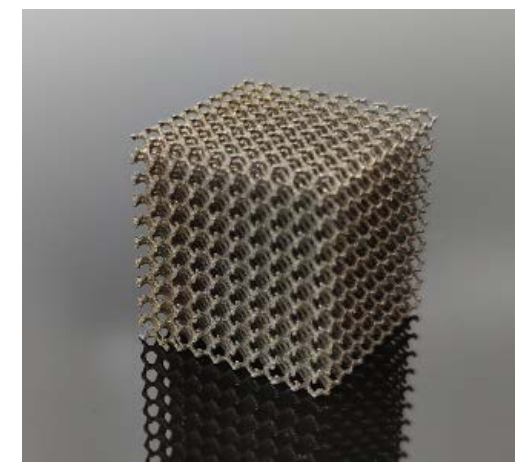

FIGURE 2: TI6AL4V BONE SCAFFOLD COMPOSED OF GYROID STRUCTURES.

Estimating the elastic modulus and yield strength of LS quickly can help designers choose a suitable structure accurately and shorten the design time of parts. In general, the elastic modulus and yield strength are calculated using the strain-stress diagram obtained from compressive experimentation on LS samples. However, fabricating all LS samples with different designed parameters to study their mechanical properties and thereby choose the most suitable structure is expensive and timeconsuming, especially since multiple candidate structures and materials are involved.

Finite element analysis (FEA) seems to be a promising method to predict the mechanical properties of LS because it only requires the 3D model of the LS. However, this method has certain disadvantages that limit its use. First, most 3D models of the LS are outputted as .stl files by modelling or programming software. These .stl files cannot be meshed directly by the simulation software; they need to be solidified first, and this process may cause the $3 \mathrm{D}$ models to lose some details of geometric features. In addition, the parameters of simulation need to be set for each 3D model, and the simulation usually takes hours. Therefore, finding a quick and accurate method to predict the mechanical properties of LS remains a challenge.

With the development of computer power, data collection, and algorithms, machine learning has been used in many areas because it can build a predictive model based on a wide variety of input features and predict the target result. Naif et al. used convolutional neural networks to predict porous media properties from 2D micro-computed tomography images [10]. Jinlong built a model to predict the permeability of porous samples from images; the results showed that, compared with FEA, this machine learning method can reduce the computational time by several orders of magnitude [11]. Therefore, machine learning offers the possibility to predict the mechanical properties of LS quickly. This paper proposes a novel method that can predict the mechanical properties of various LS and materials by machine learning. The entropy of the 3D model of LS, which represents the geometric characteristics of different LS units, together with general design parameters for different LS (such as the porosity, unit length, and elastic modulus of solid materials), are adopted as input features for the machine learning. Support vector regression (SVR) is then used to fit and predict the elastic modulus and yield strength of 57 LS models. These input features are easy to obtain, and once the predictive model is built, the prediction process is completed in a matter of seconds.

This paper presents a novel method to predict the mechanical properties of 3D printed samples composed of different LS and materials. The related literature is presented in section 2, and the input features and prediction method are introduced in section 3 . The evaluation of the predictive model and the comparison of measured and predicted values of elastic modulus and yield strength of LS samples are discussed in section 4. Conclusions and prospects for future studies are outlined in section 5 .

\section{LITERATURE REVIEW}

\subsection{Prediction method of mechanical properties of LS}

Elastic modulus and yield strength were calculated from the strain-stress diagram, based on the compressive experiments. The compressive experiment is the most basic and accurate method for investigating the mechanical properties of LS. Sing et al. studied the mechanical properties of Ti6Al4V LS in different orientations and densities [12]. FEA is a common method for predicting the mechanical properties of complex models. Maskery et al. compared the mechanical properties of gyroid, diamond, and primitive LS through both experimental and simulated methods: their results showed that the error of elastic modulus ranged from $4 \%$ to $18 \%$ [13]. However, the prediction accuracy of FEA fluctuated because the LS were too complex and there were some manufacturing defects in the asbuilt samples. Arun et al. studied the mechanical properties of six porous scaffolds by experimental and simulated methods: the 
best-predicted errors for elastic modulus and yield strength were $19.6 \%$ and $24.7 \%$, respectively [14]. Shuai et al. built and studied (by FEA) the mechanical properties of five gyroid structures with $75.1 \%$ to $88.8 \%$ porosities; prediction accuracy ranged from $30 \%$ to $56 \%$ [9]. Kevin et al. investigated the mechanical properties of seven strut structures through compressive experiments and predicted them using simulated and analytical methods; the highest predicted error could reach 300\% to $400 \%$ [15].

The experimental and simulated method is not only expensive but also time-consuming. Other researchers have proposed fitting formulae: Maxwell et al. built a multiple linear regression model to predict the mechanical properties of stochastic lattice structures in terms of density, fabric, and eigenvalue. For elastic modulus, the off-axis properties ranged from $4.2 \%$ to $13 \%$, and the coefficient of determination $\mathrm{R}^{2}$ ranged from 0.84 to 0.97 ; for yield strength, the relative error ranged from $5.1 \%$ to $10 \%$, and $\mathrm{R}^{2}$ ranged from 0.84 to 0.94 [16]. Matteo et al. used the Gibson-Ashby equation to study the relationship between the mechanical properties of LS and solid materials [17]: the $\mathrm{R}^{2}$ values were all greater than 0.98 . Although it is quick to calculate elastic modulus by fitting functions in this way, the function is only suitable for one structure and has limitations for predicting various structures. Furthermore, Han et al. investigated the mechanical properties of strut-based structures by structural mechanics analysis [18], but this only proved suitable for simple strut structures and not for complex LS such as TPMS.

\subsection{Machine learning application in mechanical properties prediction}

With its development and successful application in different areas, machine learning has attracted the attention of many researchers. Hany et al. used the shallow neural network, deep neural network, and deep learning neural network to predict the mechanical properties of the diamond lattice structure; the best mean percentage errors of elastic modulus and yield strength were $14.6 \%$ and 5.26\%, respectively [19]. However, the authors only use strut length, diameter, and orientation angle as study features; these features are not suitable for other kinds of structures. Mark et al. developed an adaptive neural networkbased model to predict femoral neck strains and fracture loads. Their results were better than the finite element model, with the $\mathrm{R}^{2}$ ranging from 0.84 to 0.98 [20]. Meng et al. predicted lumbar vertebral strength through a general regression neural network and SVR according to the grayscale distribution of quantitative computed tomography images, structural rigidity, and other features [21]. Zhenghua et al. used the chemical composition and porosity of compacts as descriptors to predict the mechanical properties of $\mathrm{Cu}-\mathrm{Al}$ alloys. Six algorithms were introduced, of which SVR showed the best prediction ability [22]. Together, these studies show the great application potential for machine learning. In the context of this study, SVR was chosen to predict the mechanical properties of LS.

\subsection{Geometric feature selection}

Bael et al. investigated the influence of geometry on the mechanical properties of LS. Their results showed that the shapes of LS will significantly affect the mechanical properties of parts. Parts composed of LS were arrayed by the LS units; thus, the mechanical properties and geometric features of LS can be represented by the single unit model, and the geometric features of unit $3 \mathrm{D}$ models were considered as the studied features in this research.

In general, geometric features such as point cloud [23,24], feature curves [25,26], and voxelisation [27] have been applied in parts retrieval and classification. Wei et al. voxelised and calculated the entropy of 3D models to represent and retrieve different machine parts [28], they all be proved as the promising methods to represent the geometric features of $3 \mathrm{~d}$ models. However, the point cloud method will generate tens of thousands of coordinate data for each 3d model, and the complex internal shapes of LS cannot be perfectly represented by the feature curves method. Thus, entropy vectors of LS unit 3D models are applied as the input parameters of the prediction model. Furthermore, Maskery et al. studied a series of $78 \%$ porosity gyroid parts with different unit lengths (from $3 \mathrm{~mm}$ to $9 \mathrm{~mm}$ ) and indicated that unit length would affect the mechanical properties of parts [29]. Bartolomeu et al. studied the elastic modulus of lattice structures with different porosities ranging from $64.2 \%$ to 93.3\%; their elastic modulus ranged from 28.6 GPa to $12.4 \mathrm{GPa}$ [30]. In summary, entropy, porosity, unit length, the density of LS unit, and elastic modulus of solid materials were considered as features in this study.

\section{METHODOLOGY \\ 3.1 Entropy of 3D models}

Typically, point cloud, view-based features, and feature curves are used in parts retrieval to represent the geometric features of parts, and they all be proved as promising and effective methods. However, for 3D printed structures, applying these methods results in certain problems, such as too much data, errors caused by inconsistent viewing directions, the difficulty of representing the complex internal structure of LS, and the feature curves method cannot effectively represent the structures with the same primitive surface. Thus, considering the universality of the method to the 3d models of LS, the geometric features of different LS units could be represented by the entropy of their voxelised 3D models.

Voxelisation involves converting the 3D model to a model consisting of pixels of a specified size; the new model is located at a space with $\mathrm{R}^{3}$ resolution. There are two kinds of pixels in this space: empty and solid pixels. To calculate the entropy of a voxelised part, first, the 3D models of LS units were voxelised into 3D voxels. To avoid too much data and ensure sufficient precision, $20^{3}, 50^{3}, 100^{3}, 150^{3}, 200^{3}$, and $300^{3}$ resolution were tested. The porosities of re-built voxelised models were calculated and compared with the $3 d$ models, thus, $100 \times 100$

$\times 100$ resolution was adopted in this study. As FIGURE 3 shows, for the circle voxelised at $20 \times 20 \times 20$ resolution, 
the proportions of solid and empty voxels were defined as $\mathrm{P}_{1}$, $\mathrm{P}_{2}$, respectively. Then, the entropy was calculated by the equation [28]:

$$
\begin{aligned}
& H_{2}=-P_{1} \log _{2} P_{1}-P_{2} \log _{2} P_{2} \\
& P_{1}+P_{2}=1
\end{aligned}
$$

where $\mathrm{H}_{2}$ represents the entropy of the $3 \mathrm{D}$ model.

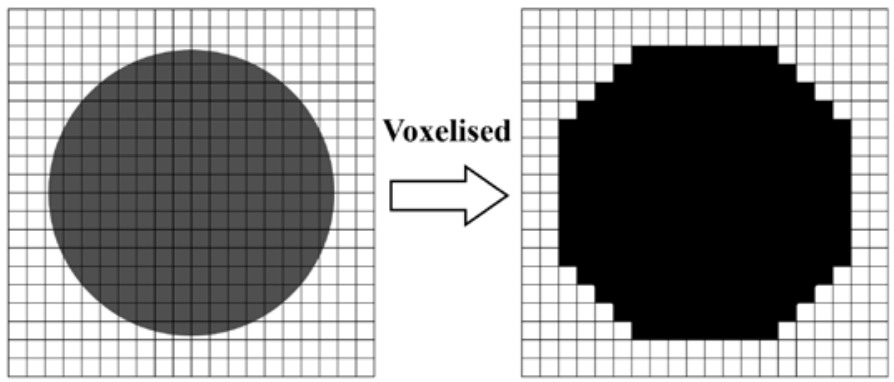

FIGURE 3: SCHEMATIC DIAGRAM OF VOXELISATION.

The global entropy of the 3D model makes it difficult to distinguish different models with the same $\mathrm{P}_{1}$ and $\mathrm{P}_{2}$ but which have different shapes. Thus, the voxelised models were divided into 100 layers. To maintain consistency, the fabricated direction $\mathrm{z}$-axis was applied as the divided direction since the compression experiments were processed in the same direction. As FIGURE 4 shows, 20 subspaces with $100 \times 100 \times 5$ resolution were divided from each voxelised model, meaning that every five layers were divided into a subspace. The $\mathrm{H}_{2}$ value of each subspace was then calculated, and an entropy vector composed of 20 entropy was obtained to represent the 3D model of the LS unit. The entropy vectors of all samples were obtained using this method and applied as the 1 to 20 input features of the predictive model.

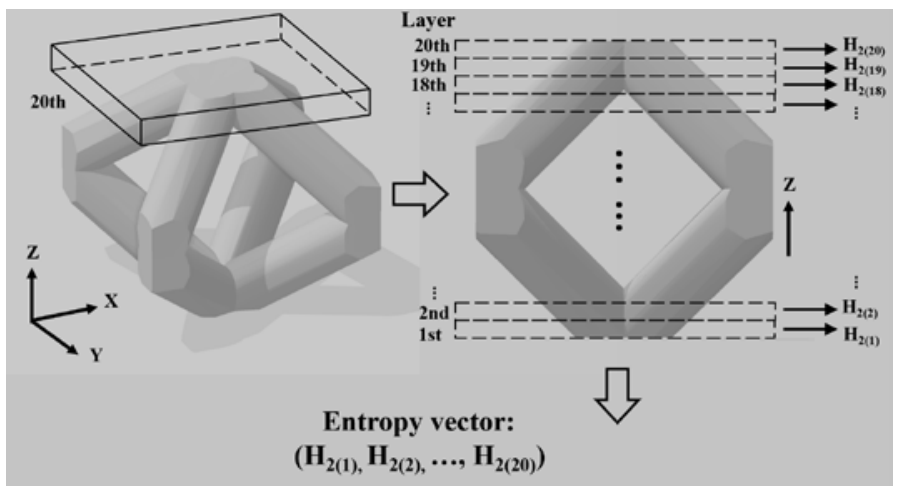

FIGURE 4: PROCESS OF DIVIDING SUBSPACE AND CALCULATING ENTROPY VECTOR.

\subsection{Design parameters of lattice structures}

Modelling the 3D models of LS units is the first step in designing parts composed of porous structures. Once the type of lattice structure is chosen, some parameters can still be modified to obtain different unit cells. For strut-based structures, as shown in FIGURE 5 (a), the length and diameter of struts were used as the featured parameters. For surface-based structures (one kind of TPMS), as shown in FIGURE 5 (b), the pore size and thickness of the surface were applied.

However, to predict the mechanical properties of different structures using one predictive model, common parameters that suit all kinds of LS must be considered in this study. As FIGURE 5 (c) shows, L is the length of the unit; another common parameter is porosity $(\mathrm{P})$, as defined by the equation below:

$P=\left(1-\frac{V_{\text {solid }}}{V_{\text {cube }}}\right) \times 100 \%$

where $\mathrm{V}_{\text {solid }}$ and $\mathrm{V}_{\text {cube }}$ are the volumes of the unit and the cube, respectively.

These two common parameters are suitable for all LS. Furthermore, for the prediction of mechanical properties of LS manufactured with different materials, the density and elastic modulus of solid metallic materials were also introduced as input features in the machine learning model.

In summary, a total of 24 parameters were used as input features: 20 entropy of subspaces, plus unit length, unit porosity, and density and elastic modulus of materials.

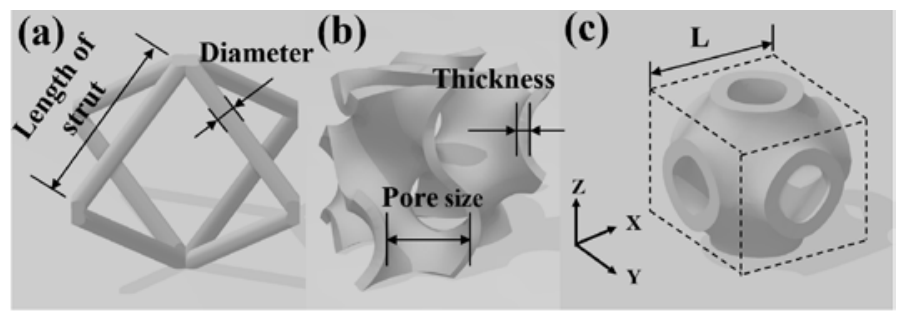

FIGURE 5: FEATURED PARAMETERS OF (a) STRUT STRUCTURES, (b) TPMS STRUCTURES, (c) COMMON PARAMETERS OF ALL LATTICE STRUCTURES.

\subsection{Collection of study samples}

Considering that the information of structures given in the related papers is not complete as input features. To obtain the complete data and correct 3d models, the details of fifty-seven SLM samples (fabricated using Ti6Al4V and 316L stainless steel powders) were collected from previous studies of the 3D printing research group, Chongqing University. The 3D models of all LS units were re-built and outputted as .stl files using Rhino software. Magics software was then used to convert all 3D models to the same accuracy of the triangular patch $(0.05 \mathrm{~mm})$ in order to eliminate the influence of modelling accuracy. To allow the predictive model to examine as many kinds of structures as possible, 11 kinds of common LS units (with different designed parameters and materials) were introduced in this study. These LS units are shown in TABLE 1 . The study set included strut structures and strut-based and sheet-based TPMS structures.

To build the predictive model, 10 samples were randomly picked from the 57 study samples to compose the test set. The remaining samples were used as the training set. 


\subsection{Algorithm and evaluation of prediction}

SVR was used as the machine learning algorithm in this study. The grid search method and 10 -fold cross-validation were conducted to obtain a robust predictive model. The predictive model was fitted using Pycharm software and the scikit-learn toolkit. The program was processed on Surface Pro 6 (Microsoft Corporation, i5-8350U, 8G RAM).

TABLE 1: CATEGORIES OF STUDIED LS SAMPLES

\begin{tabular}{|c|c|c|c|}
\hline $\begin{array}{c}\text { Category } \\
\text { number }\end{array}$ & 3D model & Structure type & $\begin{array}{l}\text { Number } \\
\text { of } \\
\text { samples }\end{array}$ \\
\hline
\end{tabular}

1

2

3

4

5

6

7

8

9

10

11
BCC

BCCZ

Strut-based

Schwarz

primitive

Strut-based

diamond

Strut-based gyroid

Strut-based

diamond

Sheet-based gyroid

Sheet-based

Schwarz

primitive

Sheet-based IWP

Neovius

FCC
3

7

3

8

3

9

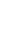

4
As FIGURE 6 shows, the 3D models of LS units were voxelised and divided into 20 subspaces; the entropy of each subspace was calculated to obtain the entropy vector, which was then combined with other studied features as input parameters to train the predictive model (processed by SVR). The root mean squared error (RMSE) and determination $\left(\mathrm{R}^{2}\right)$ were introduced to evaluate the predictive model as the following equations:

$R M S E=\sqrt{\frac{1}{m} \sum_{i=1}^{m}\left(y_{i}-\hat{y}_{i}\right)^{2}}$

$R^{2}=1-\frac{\sum_{i=1}^{m}\left(\hat{y}_{i}-y_{i}\right)^{2}}{\sum_{i=1}^{m}\left(\bar{y}_{i}-y_{i}\right)^{2}}$

where $\mathrm{m}$ is the number of samples, $\mathrm{y}_{\mathrm{i}}, \hat{\mathrm{y}}_{\mathrm{i}}$, and $\overline{\mathrm{y}}$ represent the actual, predicted and the average value of output. Furthermore, the predicted error (e) and relative error $\left(\mathrm{e}_{\mathrm{r}}\right)$ between predictive and experimental mechanical properties are defined using the following equations:

$e=\left|E_{\text {pre }}-E_{\text {exp }}\right|$

$e_{r}=\frac{\left|E_{p r e}-E_{\text {exp }}\right|}{E_{\exp }} \times 100 \%$

where $E_{\text {pre }}$ and $E_{\exp }$ represent the predicted and experimental mechanical properties (elastic modulus and yield strength) of LS, respectively.

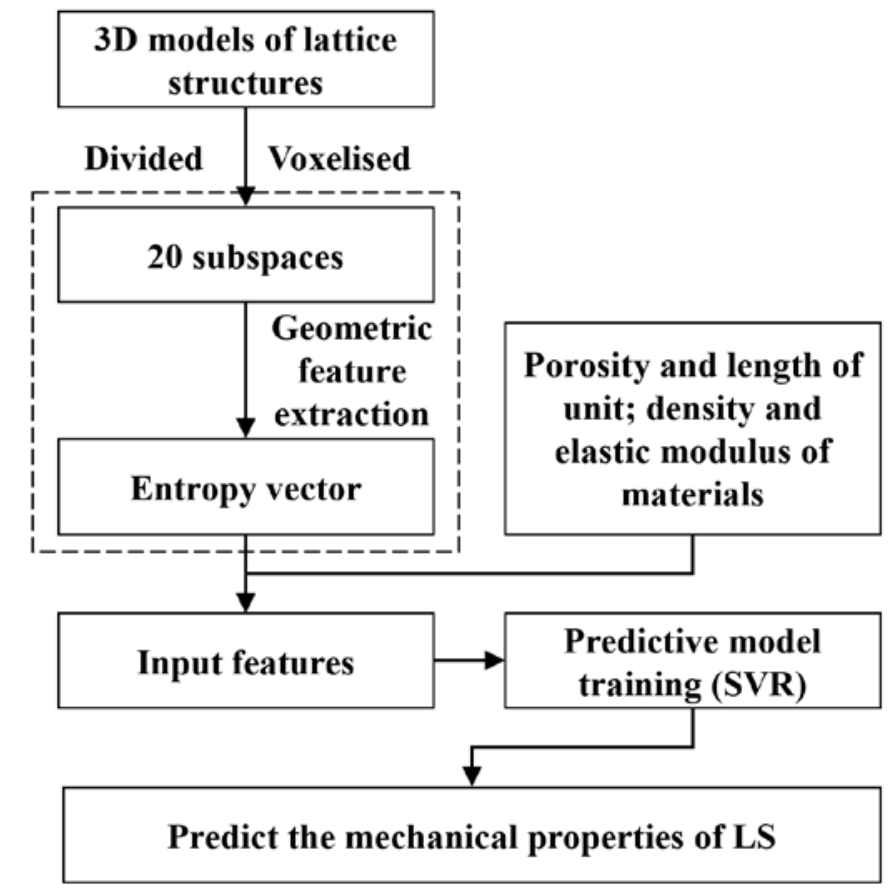

FIGURE 6: PROCESS OF PREDICTING MECHANICAL PROPERTIES OF LS UNITS BY MACHINE LEARNING. 
Also, considering the time cost of the fitting formulae and structural mechanics analysis is hard to measure, and FEA is the most common method to predict the mechanical properties of LS, this study compared the current and FEA methods to evaluate the speed and accuracy of this method.

\section{RESULTS AND DISCUSSION}

To assess whether entropy vectors can effectively represent the geometric features of different structures, TABLE 2 shows the entropy distribution of four kinds of LS with different design parameters. For the entropy distributions of different LS categories, the shapes of the distribution are significantly different. However, the 3D models in the same row belong to one kind of structure but with different design parameters, such as diameter and porosity; their entropy distributions have the same shape but different values. The results indicate that the entropy vectors are suitable for representing various lattice structures; therefore, they provide a good group of input features for the machine learning model.

TABLE 2: RESULTS OF ENTROPY DISTRIBUTIONS OF DIFFERENT LS UNITS

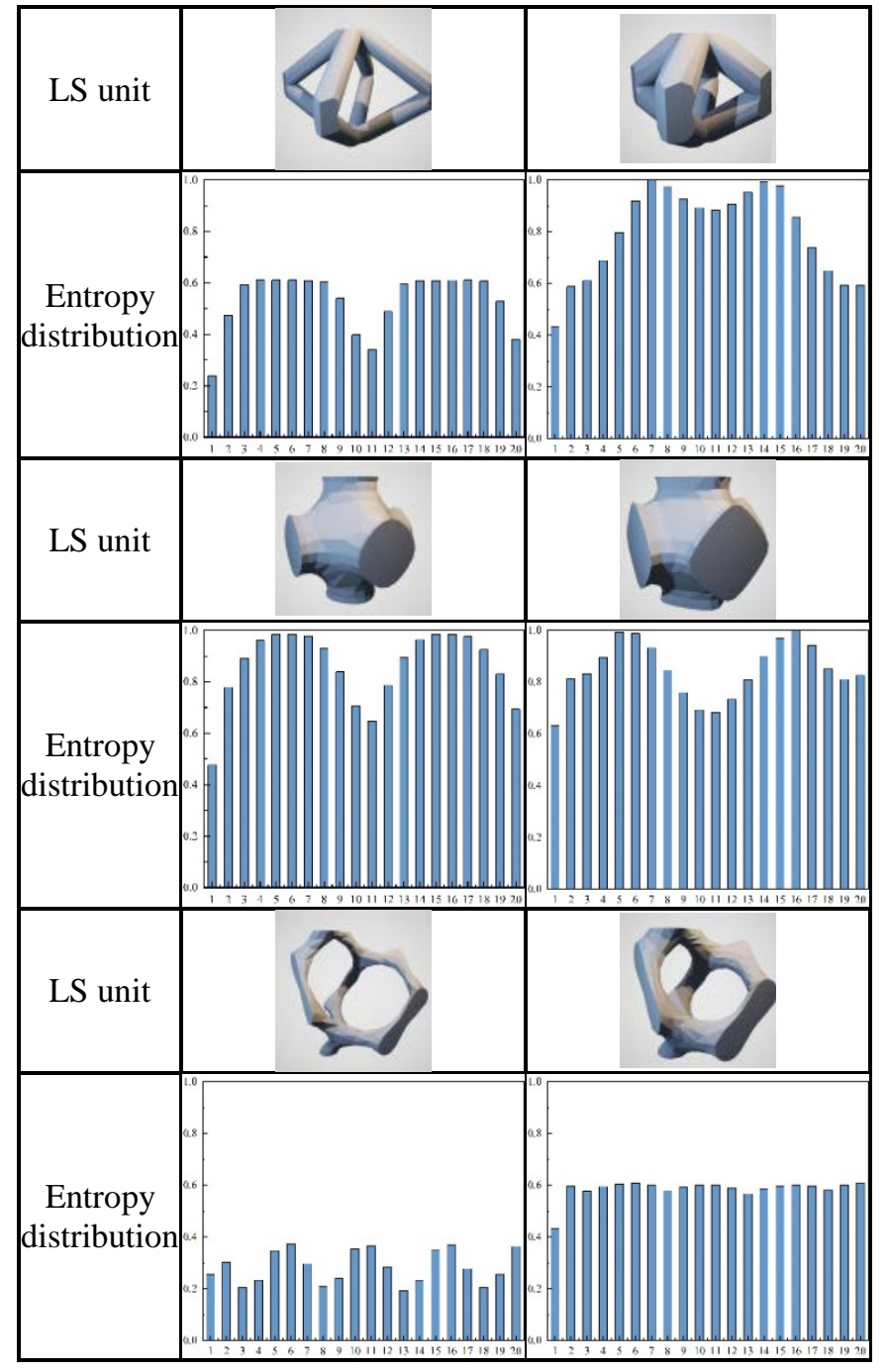

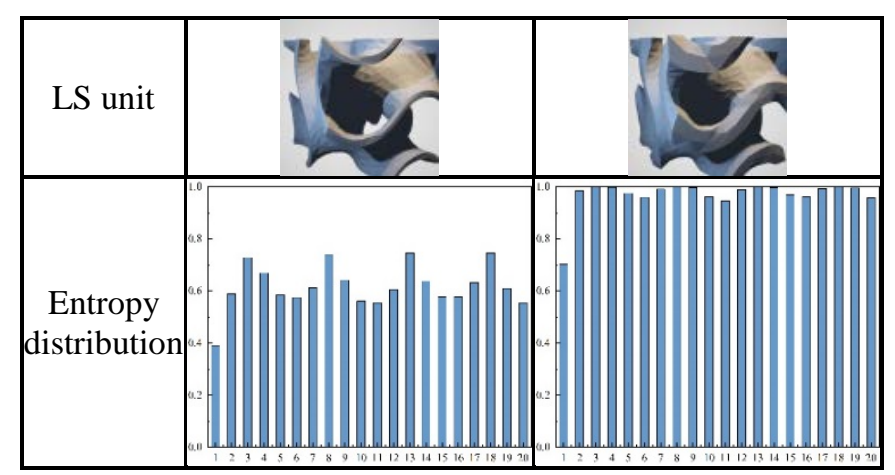

The parameters of the predictive model were optimised using the grid search method. The evaluation of the resulting model is shown in TABLE 3. For elastic modulus, in the training set, the RMSE and $\mathrm{R}^{2}$ reached 636 and 0.93 , respectively. The results also indicate that the geometric features of LS 3D models have a high correlation with elastic modulus. Considering that the actual elastic modulus of the training set ranged from $68 \mathrm{MPa}$ to $9,309 \mathrm{MPa}$, with $244 \mathrm{MPa}$ predicted error and $5.6 \%$ relative error, the results are good for this model. In the test set, the RMSE (885) is higher than in the training set. $\mathrm{R}^{2}$ is 0.81 , which is slightly lower than in the training set, and the relative error reaches $24.6 \%$. Compared to the existing prediction methods outlined in section 2.1, their $\mathrm{R}^{2}$ values ranged from 0.84 to 0.98 and relative error from $4 \%$ to $18 \%$. The results of this study show that the current prediction method has great application potential.

In terms of the yield strength predictive model, the actual yield strength of all samples ranged from 1.9 MPa to 590.3 MPa. In the training set, the RMSE and mean error were $25.96 \mathrm{MPa}$ and 14.14 MPa, respectively, and the $\mathrm{R}^{2}$ value reached 0.96 , which demonstrates a stronger correlation than the elastic modulus; however, the mean relative error was $20.1 \%$. The RMSE and $\mathrm{R}^{2}$ of the test set were worse than in the training set. Furthermore, the mean relative error is the highest at $40.9 \%$; the reasons will be analysed below.

TABLE 3: EVALUATION OF THE PREDICTIVE MODEL IN TRAINING SET AND TEST SET

\begin{tabular}{cccccc}
\hline & Set & RMSE & $\mathrm{R}^{2}$ & $\begin{array}{c}\text { Mean error } \\
(\mathrm{MPa})\end{array}$ & $\begin{array}{c}\text { Mean } \mathrm{e}_{\mathrm{r}} \\
(\%)\end{array}$ \\
\hline $\begin{array}{c}\text { Elastic } \\
\text { modulus }\end{array}$ & Training set & 636.48 & 0.93 & 244.11 & 5.66 \\
& Test set & 885.70 & 0.81 & 593.72 & 24.61 \\
& Training set & 25.96 & 0.96 & 14.14 & 20.1 \\
$\begin{array}{c}\text { Yield } \\
\text { strength }\end{array}$ & Test set & 51.74 & 0.80 & 37.14 & 40.9 \\
\hline
\end{tabular}

FIGURE 7 (a) shows the actual and predicted elastic modulus of the training set. Most of the predicted values have a 
strong correlation with the actual results. The largest predicted error occurs in sample 22, a sheet-based I-WP structure with $55 \%$ porosity; the error is 3,007 MPa. This may be because I-WP structures have greater mechanical properties compared with other structures, and this error could be reduced by introducing more samples with different parameters.

FIGURE 7 (b) shows the relative errors for the training set. The largest relative error (47.9\%) is observed in sample 8 , an $85 \%$ porosity strut-based diamond structure whose actual elastic modulus is only $1,074 \mathrm{MPa}$. Its predicted error is $514 \mathrm{MPa}$, which is slightly higher than the mean error of the training set (244 MPa) and far below the maximum error observed in sample 22.
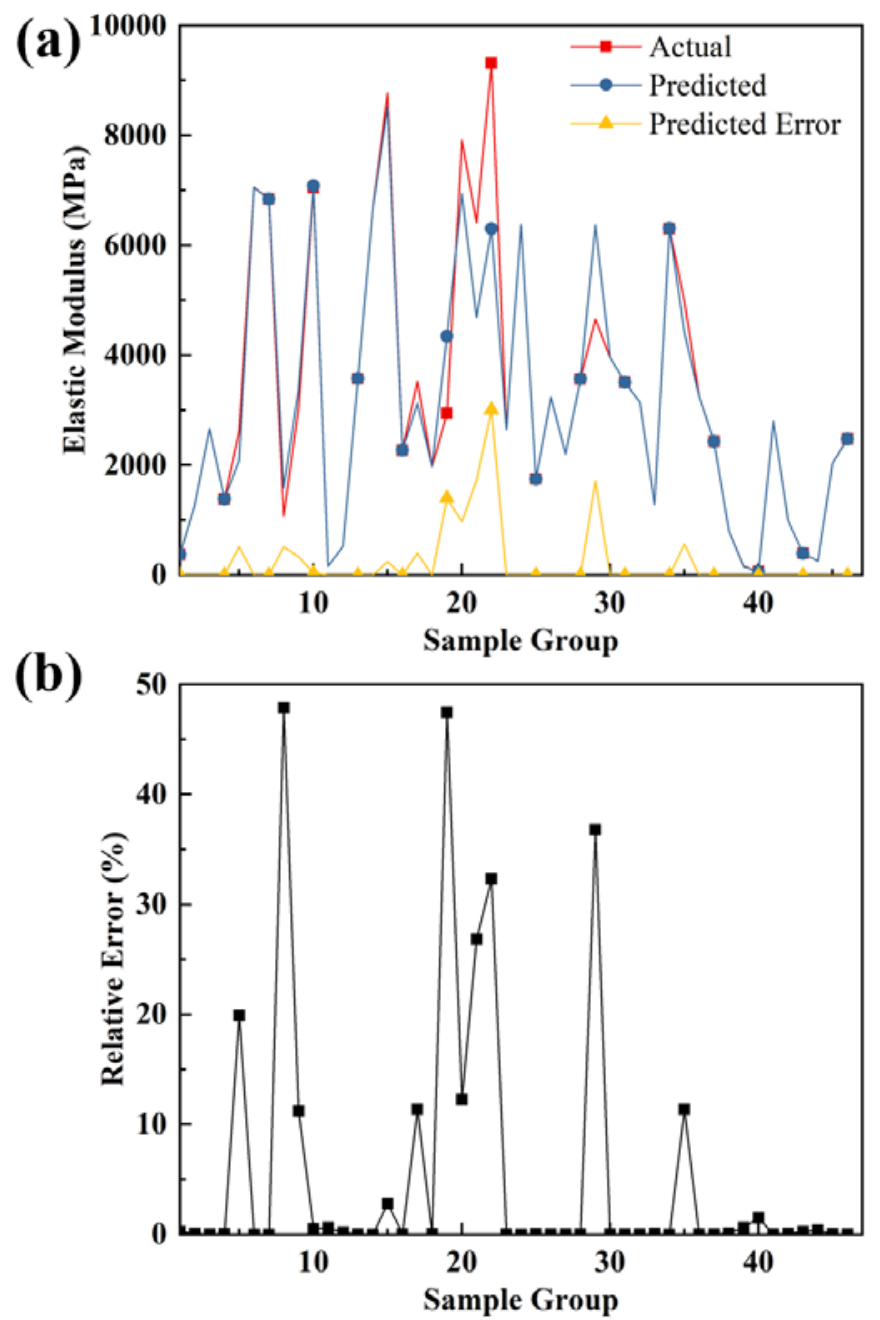

FIGURE 7: ELASTIC MODULUS OF TRAINING SET: (a) ACTUAL/PREDICTED VALUES; (b) RELATIVE ERROR.

To compare the actual and predicted results, 10 samples in the test set were inputted into the predictive model; the results are shown in FIGURE 8. The largest errors were observed in samples 2 and 7, which have roughly 55\% porosity and belong to strut-based and sheet-based Schwarz primitive structures, respectively. The possible reason for the error is that the elastic modulus of LS will increase significantly as porosity decreases, and the porosities of 44 of 57 samples were higher than $60 \%$. With more lower-porosity samples, the predicted results should show great improvement. For relative error, only sample 10 has $37.5 \mathrm{MPa}$ actual elastic modulus, which will make the relative error sensitive to the predicted difference.

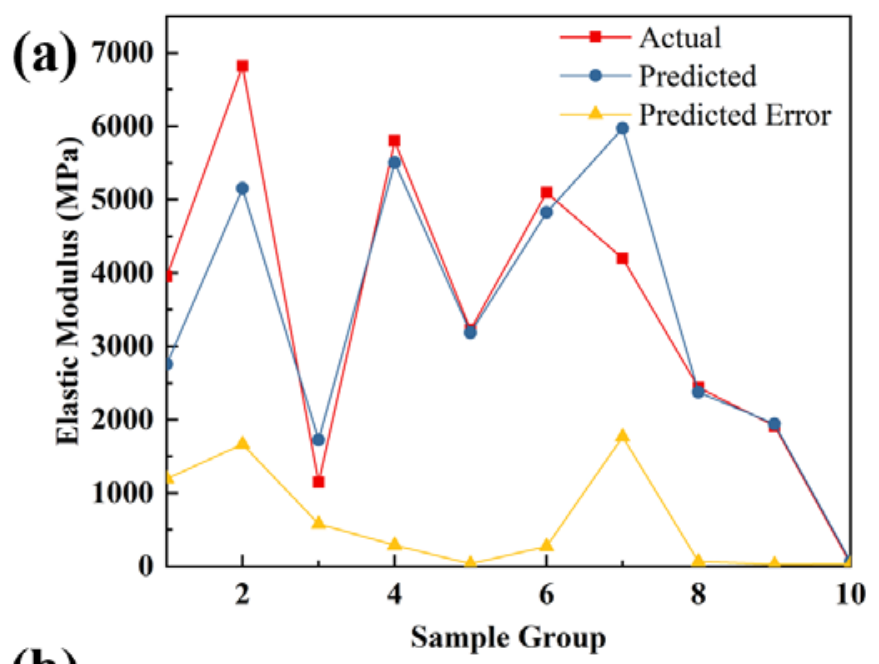

(b)

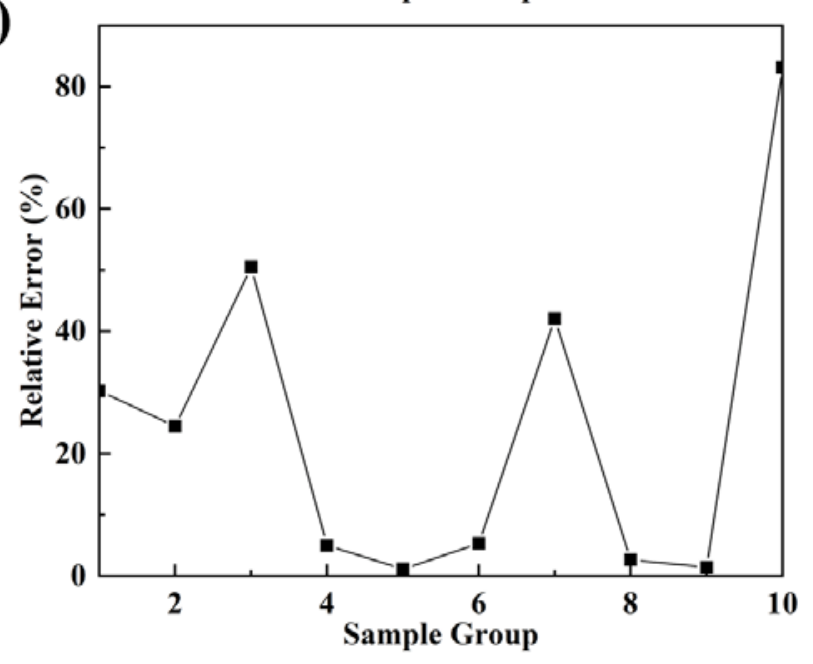

FIGURE 8: ELASTIC MODULUS OF TEST SET: (a) ACTUAL/PREDICTED VALUES; (b) RELATIVE ERROR.

FIGURE 9 shows the differences between actual and predicted yield strengths. Generally, the predicted curve matched the actual curve well. The minimum predicted error is $0.97 \mathrm{MPa}$, while the maximum predicted error is $118.23 \mathrm{MPa}$. For sample 10 , the strut-based diamond structure, the actual value is 249.5 MPa. Except for three samples with high predicted errors, the predicted errors of the other 44 samples were lower than 23.5 $\mathrm{MPa}$. Four relative errors are high, while the relative errors of the 43 remaining samples are lower than $27 \%$. The highest value is $164 \%$ : the relative error of sample 11 , a strut-based gyroid structure, which has $95 \%$ porosity and 6.1 yield strength. However, the predicted error of sample 11 is only $10 \mathrm{MPa}$, lower 
than the mean error of $14 \mathrm{MPa}$. The mean relative error would reach $26.4 \%$ by excluding sample 11 .
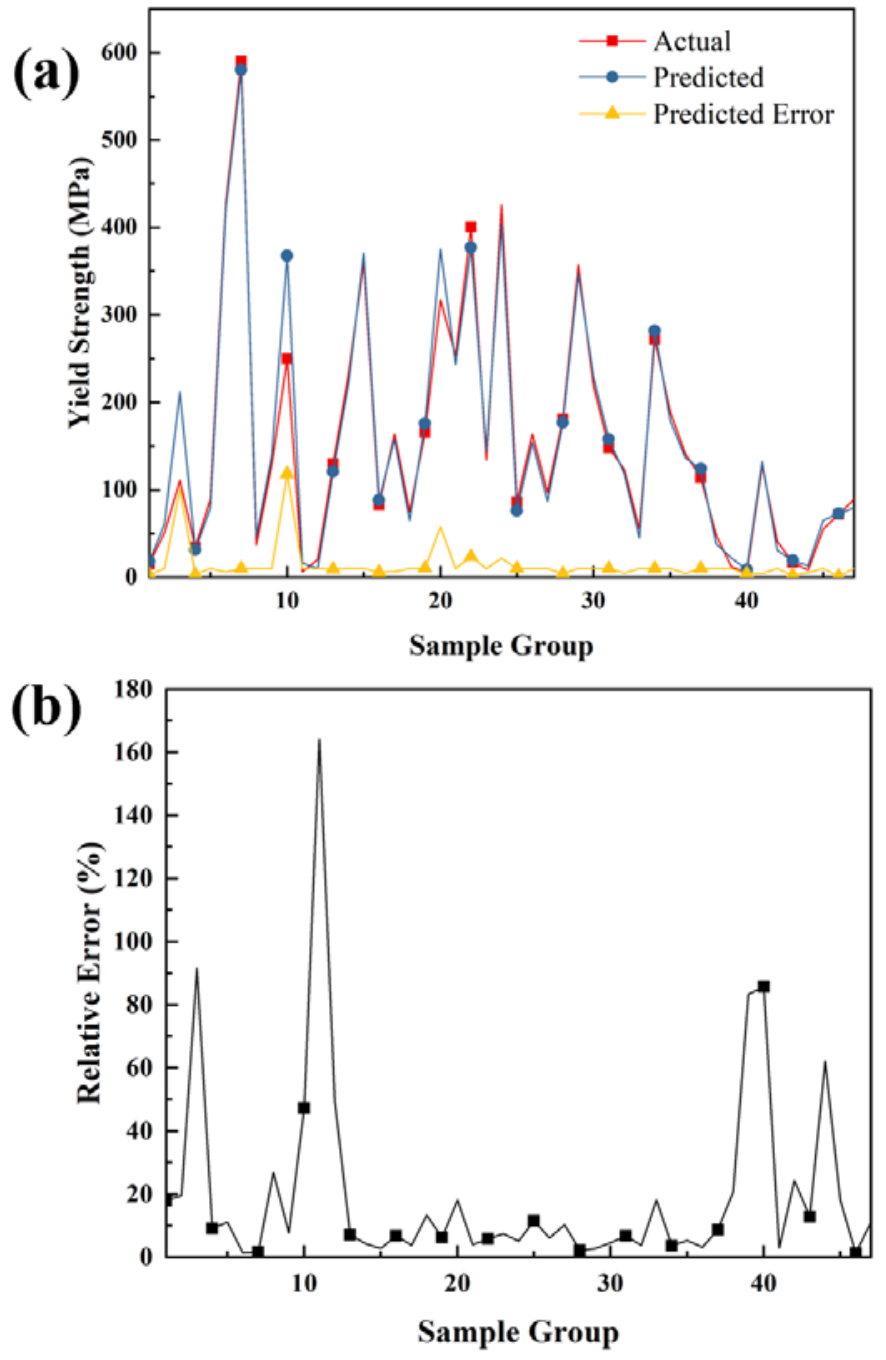

FIGURE 9: YIELD STRENGTH OF TRAINING SET: (a) ACTUAL/PREDICTED VALUES; (b) RELATIVE ERROR.

The maximum predicted error of the test set is $128.77 \mathrm{MPa}$, and the actual value of this sample is $326 \mathrm{MPa}$. The errors for 8 out of 10 samples are lower than $40 \mathrm{MPa}$. As FIGURE 10 (b) shows, the relative error of sample 10 is $171 \%$, but the predicted error is only 3.2 MPa, which has a significant effect on the mean relative error; if sample 10 is excluded, it would decline to $26.4 \%$.

Considering that even the mechanical properties obtained from the compression experiments have fluctuating errors, as TABLE 4 shows, errors ranged from $33 \mathrm{MPa}$ to $162 \mathrm{MPa}$, while the elastic modulus ranged from 1,465 $\mathrm{MPa}$ to 2,676 $\mathrm{MPa}$ [31]. Experimental error ranged from $100 \mathrm{MPa}$ to $130 \mathrm{MPa}$, and experimental elastic moduli of LS ranged from 2,700 MPa to 3,600 MPa [32]. Furthermore, errors from $120 \mathrm{MPa}$ to 3,640 MPa for elastic modulus and $0.38 \mathrm{MPa}$ to $12 \mathrm{MPa}$ for yield strength have also been reported [15]. Thus, the predicted errors of $244 \mathrm{MPa}$ to $593 \mathrm{MPa}$ for elastic modulus and $14.14 \mathrm{MPa}$ to 37.14 MPa for yield strength in this study still show good agreement, since the elastic modulus and yield strength ranged from $37.5 \mathrm{MPa}$ to $9,309 \mathrm{MPa}$ and $1.9 \mathrm{MPa}$ to $590.3 \mathrm{MPa}$, respectively.
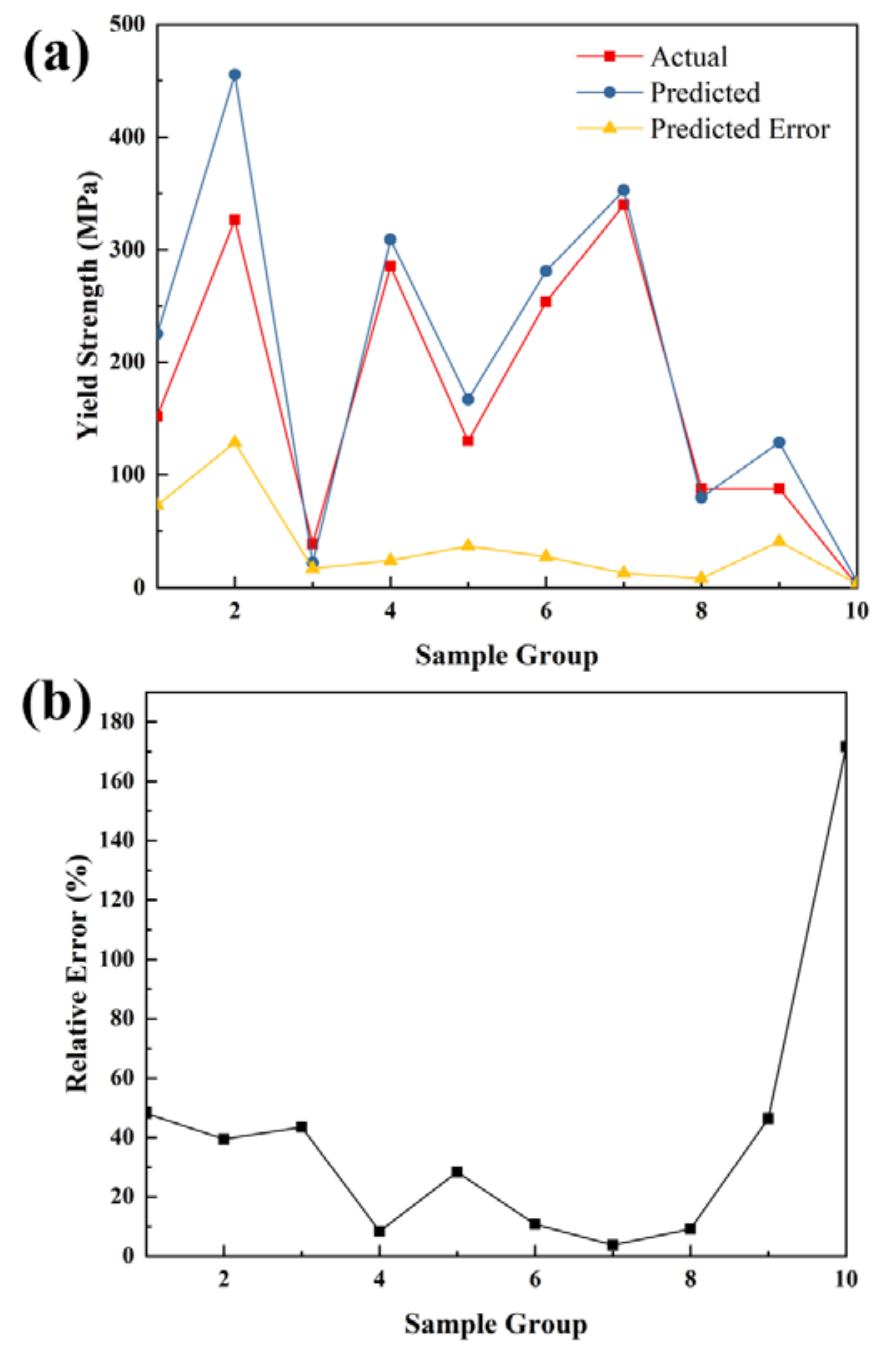

FIGURE 10: YIELD STRENGTH OF TEST SET: (a) ACTUAL/PREDICTED VALUES; (b) RELATIVE ERROR.

As TABLE 5 shows, the results and time costs of the formula, FEA, and the current methods are compared with ref [5]. The yield strength of all samples, and the elastic modulus of complex structure FCC-BCC, can not be predicted by the formula method. FEA method exhibits the lowest error of predicted yield strength of BCC structures, while for complex fcc-BCC structure, the SVR method shows higher accuracy. For the time consumption, once the SVR model is built, the prediction will finish in about 5 secs, while FEA will cost about 30 mins in the simulated process. 
TABLE 4: COMPARISON OF ERRORS IN PREVIOUS AND CURRENT STUDIES

\begin{tabular}{ccccc}
\hline & $\begin{array}{c}\text { Elastic modulus } \\
\text { range (MPa) }\end{array}$ & $\begin{array}{c}\text { Error } \\
(\mathrm{MPa})\end{array}$ & $\begin{array}{c}\text { Yield strength } \\
\text { range (MPa) }\end{array}$ & $\begin{array}{c}\text { Error } \\
(\mathrm{MPa})\end{array}$ \\
\hline$[31]$ & $1465 \sim 2676$ & $33 \sim 162$ & - & \\
{$[32]$} & $2700 \sim 3600$ & $100 \sim 130$ & - & \\
& & $120 \sim$ & $9.3 \sim 327.47$ & $0.38 \sim 12$ \\
{$[15]$} & $1060 \sim 28590$ & 3640 & & \\
& & & & \\
{$[33]$} & $2700 \sim 7400$ & $100 \sim 400$ & $233 \sim 520$ & $3 \sim 60$ \\
$\begin{array}{c}\text { Current } \\
\text { study }\end{array}$ & $37.5 \sim 9309$ & $244 \sim 593$ & $1.9 \sim 590.3$ & $14.14 \sim$ \\
\hline
\end{tabular}

TABLE 5: COMPARISON OF FORMULA, FEA, AND CURRENT METHODS

\begin{tabular}{|c|c|c|c|c|c|c|}
\hline & \multicolumn{3}{|c|}{$\begin{array}{l}\text { Error of elastic } \\
\text { modulus (MPa) }\end{array}$} & \multicolumn{3}{|c|}{$\begin{array}{c}\text { Error of yield } \\
\text { strength (MPa) }\end{array}$} \\
\hline & $\begin{array}{c}\text { Form } \\
\text { ula }\end{array}$ & FEA & SVR & $\begin{array}{l}\text { For } \\
\text { mula }\end{array}$ & FEA & SVR \\
\hline$\overline{B C C} 1$ & 117 & 521 & 1 & - & 8 & 10 \\
\hline BCC 2 & 23 & 60 & 1 & - & 2.5 & 10 \\
\hline BCC 3 & 24.5 & 67.5 & 1 & - & 1.15 & 4.03 \\
\hline BCC 4 & 19.5 & 10.5 & 31 & - & $\begin{array}{c}0.37 \\
5\end{array}$ & 3.26 \\
\hline $\begin{array}{c}\text { FCC- } \\
\text { BCC } 1\end{array}$ & - & 2200 & 1 & - & 14 & 3.81 \\
\hline $\begin{array}{c}\text { FCC- } \\
\text { BCC } 2\end{array}$ & - & 407 & 1 & - & 13.5 & 10 \\
\hline $\begin{array}{c}\text { FCC- } \\
\text { BCC } 3\end{array}$ & - & 170 & 1 & - & 4.5 & 2.2 \\
\hline $\begin{array}{c}\text { FcC- } \\
\text { BCC } 4\end{array}$ & - & 56 & 1 & - & 1.1 & 5.03 \\
\hline $\begin{array}{l}\text { Averag } \\
\text { e time }\end{array}$ & - & $\begin{array}{l}\sim 30 \\
\text { mins }\end{array}$ & $\begin{array}{l}\sim 5 \\
\text { secs }\end{array}$ & - & $\begin{array}{l}\sim 30 \\
\text { mins }\end{array}$ & $\begin{array}{l}\sim 5 \\
\text { secs }\end{array}$ \\
\hline
\end{tabular}

In summary, the predictive model in this study shows the potential to predict the mechanical properties of 3D printed structures. The study also proves that geometric features represented by entropy vectors have a strong correlation with mechanical properties in LS since $\mathrm{R}^{2}$ ranges from 0.8 to 0.96 . The highest accuracy of the predictive model can also reach the level reported by previous studies. Furthermore, this method has the following advantages:

(1) The model can predict the mechanical properties of one LS unit in a matter of seconds.

(2) The model is suitable for different types of structures and predicts the mechanical properties of LS made of different materials.

(3) The model exhibits the potential to predict other properties of LS, such as permeability and failure mode.

\section{CONCLUSIONS}

To investigate an effective method to predict the mechanical properties of LS, this study proposed a novel method based on machine learning that extracts the entropy vector from LS unit 3D models to represent the geometric features of LS, in combination with other commonly designed parameters as input features. The predictive model was then built using SVR. The results include the following:

(1) Entropy vectors can effectively represent the geometric features of LS. Similar shapes of entropy distributions are observed in the same types of structures, while the distribution shape varies between different types of structures; dividing the subspaces along the compression direction can eliminate the differences caused by the random dividing direction.

(2) This study collected 57 LS samples, and the model to predict the elastic modulus of LS was successfully built based on SVR. For elastic modulus, RMSE was measured at 636.48 and $\mathrm{R}^{2}$ at 0.93 for the training set; for the test set, RMSE was 885.7 and $\mathrm{R}^{2}$ was 0.81 . For yield strength, $\mathrm{R}^{2}$ and mean predicted error ranged from 0.8 to 0.96 and $14.14 \mathrm{MPa}$ to $37.14 \mathrm{MPa}$, respectively. This indicates that the chosen input features have a strong correlation with the mechanical properties of LS.

(3) Compared with common predicted methods, the current method can reach the accuracy of other methods and isn't limited by materials and LS categories. In particular, the predicted time is reduced from tens of minutes to a few seconds, which can greatly improve the efficiency of the design process.

In summary, compared with the high-cost experimental method and the time-consuming simulated method, this study proposes a prediction method for the elastic modulus of LS that has genuine potential application value. It has the advantage of being applicable to various kinds of structures and materials, while other methods based on machine learning and formulae can only be applied to one kind of structure. This study is significant as it can improve the efficiency of designing lattice structures, thereby reducing time and costs in the design phase.

Future studies will consider the processing parameters of the SLM machine, the expected relative density, and the manufacturing errors of structures to enhance prediction accuracy. The study of predicting the failure modes of lattice structures will also be of interest.

\section{ACKNOWLEDGEMENTS}

This paper was supported by the National Natural Science Foundation of China (Grant No: 51975073, No. 51805052) and the China Scholarship Council (CSC). The authors would like to thank Zhihang Li and Fuyu Guo (Chongqing University) for programming assistance, Xiaojie Fan (Chongqing University) for collecting data of samples.

\section{REFERENCES}

[1] Lei H, Li C, Meng J, et al. Evaluation of compressive properties of SLM-fabricated multi-layer lattice 
structures by experimental test and $\mu$-CT-based finite element analysis[J]. Materials \& Design, 2019,169:107685.

[2] Jin N, Yan Z, Wang Y, et al. Effects of heat treatment on microstructure and mechanical properties of selective laser melted Ti-6Al-4V lattice materials[J]. International Journal of Mechanical Sciences, 2021,190:106042.

[3] Li P, Ma Y E, Sun W, et al. Fracture and failure behavior of additive manufactured Ti6Al4V lattice structures under compressive load[J]. Engineering Fracture Mechanics, 2021,244:107537.

[4] Zhang L, Feih S, Daynes S, et al. Energy absorption characteristics of metallic triply periodic minimal surface sheet structures under compressive loading[J]. Additive Manufacturing, 2018,23:505-515.

[5] Feng Q, Tang Q, Liu Y, et al. Quasi-static analysis of mechanical properties of Ti6Al4V lattice structures manufactured using selective laser melting[J]. The International Journal of Advanced Manufacturing Technology, 2018,94(5-8):2301-2313.

[6] Ma S, Tang Q, Feng Q, et al. Mechanical behaviours and mass transport properties of bone-mimicking scaffolds consisted of gyroid structures manufactured using selective laser melting[J]. Journal of the Mechanical Behavior of Biomedical Materials, 2019,93:158-169.

[7] Song J, Tang Q, Feng Q, et al. Effect of heat treatment on microstructure and mechanical behaviours of $18 \mathrm{Ni}$ 300 maraging steel manufactured by selective laser melting[J]. Optics \& Laser Technology, 2019,120:105725.

[8] Zhang L, Song B, Choi S, et al. A Topology Strategy to Reduce Stress Shielding of Additively Manufactured Porous Metallic Biomaterials[J]. International Journal of Mechanical Sciences, 2021:106331.

[9] Ma S, Tang Q, Han X, et al. Manufacturability, Mechanical Properties, Mass-Transport Properties and Biocompatibility of Triply Periodic Minimal Surface (TPMS) Porous Scaffolds Fabricated by Selective Laser Melting[J]. Materials \& Design, 2020,195:109034.

[10] Alqahtani N, Armstrong R T, Mostaghimi P. Deep learning convolutional neural networks to predict porous media properties: SPE Asia Pacific oil and gas conference and exhibition, 2018[C]. Society of Petroleum Engineers.

[11] Wu J, Yin X, Xiao H. Seeing permeability from images: fast prediction with convolutional neural networks[J]. Science Bulletin, 2018,63(18):1215-1222.

[12] Choy S Y, Sun C, Leong K F, et al. Compressive properties of Ti-6Al-4V lattice structures fabricated by selective laser melting: Design, orientation and density[J]. Additive Manufacturing, 2017,16:213-224.

[13] Maskery I, Sturm L, Aremu A O, et al. Insights into the mechanical properties of several triply periodic minimal surface lattice structures made by polymer additive manufacturing[J]. Polymer, 2018,152:62-71.

[14] Arjunan A, Demetriou M, Baroutaji A, et al. Mechanical performance of highly permeable laser melted Ti6Al4V bone scaffolds[J]. Journal of the Mechanical Behavior of Biomedical Materials, 2019:103517.

[15] Hazlehurst K, Wang C J, Stanford M. Evaluation of the stiffness characteristics of square pore CoCrMo cellular structures manufactured using laser melting technology for potential orthopaedic applications[J]. Materials \& Design, 2013,51:949-955.

[16] Munford M, Hossain U, Ghouse S, et al. Prediction of anisotropic mechanical properties for lattice structures[J]. Additive Manufacturing, 2020,32:101041.

[17] Benedetti M, Klarin J, Johansson F, et al. Study of the Compression Behaviour of Ti6Al4V Trabecular Structures Produced by Additive Laser Manufacturing[J]. Materials, 2019,12(9):1471.

[18] Han C, Yan C, Wen S, et al. Effects of the unit cell topology on the compression properties of porous CoCr scaffolds fabricated via selective laser melting[J]. RAPID PROTOTYPING JOURNAL, 2017,23(1):1627.

[19] Hassanin H, Alkendi Y, Elsayed M, et al. Controlling the Properties of Additively Manufactured Cellular Structures Using Machine Learning Approaches[J]. Advanced Engineering Materials, 2020,22(3):1901338.

[20] Taylor M, Perilli E, Martelli S. Development of a surrogate model based on patient weight, bone mass and geometry to predict femoral neck strains and fracture loads[J]. Journal of Biomechanics, 2017,55:121-127.

[21] Zhang M, Gong H, Zhang K, et al. Prediction of lumbar vertebral strength of elderly men based on quantitative computed tomography images using machine learning[J]. Osteoporosis International, 2019,30(11):2271-2282.

[22] Deng Z, Yin H, Jiang $X$, et al. Machine-learningassisted prediction of the mechanical properties of $\mathrm{Cu}$ Al alloy[J]. International Journal of Minerals, Metallurgy and Materials, 2020,27(3):362-373.

[23] Furuya T, Ohbuchi R. Learning part-in-whole relation of 3D shapes for part-based 3D model retrieval[J]. Computer Vision and Image Understanding, 2018,166:102-114.

[24] Kim H, Cha M, Mun D. Shape distribution-based approach to comparing 3D CAD assembly models[J]. Journal of Mechanical Science and Technology, 2017,31(12):5627-5638.

[25] Lu Z, Guo J, Xiao J, et al. Extracting Cycle-aware Feature Curve Networks from 3D Models[J]. Computer-Aided Design, 2021,131:102949.

[26] Makem J E, Fogg H J, Mukherjee N. Automatic Feature Recognition Using the Medial Axis for Structured 
Meshing of Automotive Body Panels[J]. ComputerAided Design, 2020,124:102845.

[27] Kim S, Chi H, Ramani K. Object Synthesis by Learning Part Geometry with Surface and Volumetric Representations[J]. Computer-Aided Design, 2021,130:102932.

[28] Wei L, Yuanjun H. Representation and retrieval of 3D CAD models in parts library[J]. The International Journal of Advanced Manufacturing Technology, 2008,36(9-10):950-958.

[29] Maskery I, Aboulkhair N T, Aremu A O, et al. Compressive failure modes and energy absorption in additively manufactured double gyroid lattices[J]. Additive Manufacturing, 2017,16:24-29.

[30] Bartolomeu F, Costa M M, Alves N, et al. Selective Laser Melting of Ti6Al4V sub-millimetric cellular structures: Prediction of dimensional deviations and mechanical performance[J]. Journal of the Mechanical Behavior of Biomedical Materials, 2021,113:104123.

[31] Ataee A, Li Y, Brandt M, et al. Ultrahigh-strength titanium gyroid scaffolds manufactured by selective laser melting (SLM) for bone implant applications[J]. ACTA MATERIALIA, 2018,158:354-368.

[32] Wang S, Liu L, Zhou X, et al. The design of Ti6Al4V Primitive surface structure with symmetrical gradient of pore size in biomimetic bone scaffold[J]. Materials \& Design, 2020,193:108830.

[33] Liao B, Xia R F, Li W, et al. 3D-Printed Ti6Al4V Scaffolds with Graded Triply Periodic Minimal Surface Structure for Bone Tissue Engineering[J]. Journal of Materials Engineering and Performance, 2021. 